\title{
Antioxidant enzymes in the developing lungs of egg-laying and metamorphosing vertebrates
}

\author{
Adam P. Starrs, Sandra Orgeig*, Christopher B. Daniels, Margaret Davies and Olga V. Lopatko \\ Department of Environmental Biology, Adelaide University, Adelaide SA 5005, Australia \\ *Author for correspondence (e-mail: sandra.orgeig@adelaide.edu.au) \\ ¥Present address: Department of Clinical and Experimental Pharmacology, Adelaide University, Adelaide SA 5005, Australia
}

Accepted 9 August 2001

\begin{abstract}
Summary
The activities of the pulmonary antioxidant enzymes (AOE), superoxide dismutase (SOD), glutathione peroxidase (GPx) and catalase, increase in the final $10-20 \%$ of gestation in the mammalian lung, to protect the lung from attack by increasing levels of reactive oxygen species at birth. Whether the increase occurs as a normal 'preparation for birth', i.e. by a genetically determined mechanism, or in response to increased levels of oxygen, i.e. in response to the environment, is not completely understood. We examined the activities of catalase, SOD and GPx in the developing lungs of two oviparous vertebrate species, the chicken (Gallus gallus) and an agamid lizard (Pogona vitticeps), and in a metamorphosing vertebrate, the anuran Limnodynastes terraereginae. During in ovo development embryos come into contact with higher levels of environmental oxygen, and at a much earlier stage of development, compared with the intrauterine development of mammals. Furthermore, in metamorphosing frogs, the lungs are inflated at an early stage to aid in buoyancy, although the gas-exchange function only develops much later upon final metamorphosis. Here, we hypothesise that the activity of the AOE will be elevated relatively much earlier during development in both oviparous and metamorphosing vertebrates. We also examined the effect of mild hypoxia (17\% oxygen) on the development of the pulmonary AOE

incubation. Catalase and SOD activities were virtually identical in hypoxic and normoxic embryos of the chicken, but GPx activity was significantly affected by hypoxia. In the developing frog, the activities of all enzymes were high at stage 30, demonstrating that the system is active before the lung displays any significant gas-exchange function. SOD and GPx activity did not increase further with development. Catalase activity increased after stage 40, presumably correlating with an increase in air-breathing. In summary, catalase expression in the two oviparous vertebrates appears to be completely under genetic control as the activity of this enzyme does not change in response to changes in oxygen tension. However, in tadpoles, catalase may be responsive to environmental oxygen. SOD also appears to follow a largely genetically determined program in all species. Under normoxic conditions, GPx appears to follow a genetically determined developmental pattern, but this enzyme demonstrated the largest capacity to respond to environmental oxygen fluctuations. In conclusion, it appears that the AOE are differentially regulated. Furthermore, the $\mathrm{AOE}$ in the different species appear to have evolved different levels of dependency on environmental variables. Finally, the late developmental increase in AOE activity seen in mammals is not as pronounced in oviparous and metamorphosing vertebrates.
\end{abstract} in the chicken, to test the hypothesis that these enzymes are responsive to environmental oxygen. In the normoxic lung of both Gallus gallus and Pogona vitticeps, catalase and GPx activities were significantly increased in late incubation, whereas SOD activity decreased in late
Key words: reptile, bird, eggs, frog, metamorphosis, catalase, glutathione peroxidase, superoxide dismutase, Gallus gallus, Pogona vitticeps, Limnodynastes terraereginae.

\section{Introduction}

The conversion of oxygen to water produces highly reactive oxygen species that are known to induce the peroxidation of polyunsaturated lipids in cell membranes and to cause degradation of structural proteins, enzymes and ribonucleic acids. These reactive intermediates of oxygen metabolism are converted mainly into molecular oxygen and water by antioxidant enzymes and normally are kept to a minimum in blood and organ tissues (Fridovich, 1978; Fridovich and Freeman, 1986). There are three main antioxidant enzymes. Superoxide dismutase (SOD) catalyses the conversion of the superoxide radical to hydrogen peroxide and oxygen (McCord and Fridovich, 1969). Catalase converts hydrogen peroxide to molecular oxygen and water (Fridovich, 1978). Glutathione peroxidase (GPx) catalyses the same reactions as catalase and 
also converts products of lipid peroxidation back into functional membrane lipids (Chiu et al., 1976; Frank and Massaro, 1980).

For mammals, birth leads to an immediate dramatic increase in the exposure of the lung to oxygen, from approx. $25-100 \mathrm{mmHg}(1 \mathrm{mmHg}=133.3 \mathrm{~Pa})$. This phenomenon is known as 'relative hyperoxia' and is accompanied by an increase in reactive oxygen species (Rickett and Kelly, 1990). The lung responds to an increased production of reactive oxygen species during this period by increasing the activity of the antioxidant enzymes (AOE). An increase in all three AOEs has been demonstrated in the lungs of most laboratory mammals, including the rat, rabbit, guinea pig, mouse, hamster and sheep. However, most of these increases begin to occur before birth, in particular in the last 10-20\% of the gestation period (Chen and Frank, 1993; Frank and Groseclose, 1984; Frank and Sosenko, 1987; Gerdin et al., 1985; Rickett and Kelly, 1990; Tanswell and Freeman, 1984; Walther et al., 1991).

Oviparous species have an entirely different method of parturition, which may be accompanied by differences in the developmental pattern of AOEs. Oviparous embryos, such as the chicken, obtain their oxygen from an air-cell within the egg-space, which is in direct contact with the outside environment (Starck, 1998). Hence, egg-dwelling embryos may be more susceptible to the effects of external conditions and may experience higher pulmonary oxygen tensions than viviparous embryos. In the embryo of the chicken, Gallus gallus, for example, the oxygen tension in pulmonary venous blood falls from $71 \mathrm{mmHg}$ to $49 \mathrm{mmHg}$ between day 14 and day 19 (of a 21-day incubation) (Freeman and Misson, 1970). Therefore, in early incubation, the chick embryo is exposed to considerably higher oxygen tensions than the mammalian foetus (about $25 \mathrm{mmHg}$ ). The AOE system in the chicken and other oviparous species may therefore be considerably more advanced during the first two-thirds of incubation than in mammals, due simply to the increased prenatal exposure of the foetus to reactive oxygen species. Hence, the late gestational increase in AOE activity typically seen in mammals may not occur in oviparous species.

Only two studies have examined the AOEs in the lungs of non-mammalian embryos over the last $20-30 \%$ of the incubation period, both in chickens, and both with contradictory results. Van Golde and coworkers measured catalase, SOD and GPx activity in the lungs of chicken embryos from day 10 until hatching (Van Golde et al., 1998). SOD activity peaked at day 18 , then remained constant till hatching; GPx activity peaked at day 16 and declined to hatching; and catalase activity peaked at pipping (day 20). Pipping is the stage immediately prior to hatching, where the embryo has broken through the shell membrane but not the shell, and has commenced air-breathing in the air-cell. Surai measured the same enzymes in chicken lungs from 15 days of incubation until hatching (Surai, 1999). SOD activity decreased from day 15 till hatching, whereas GPx activity reached its lowest point at day 19 (pip activity not determined) and increased thereafter until hatching. Catalase activity remained relatively unchanged throughout late incubation with a slight increase from day 19 to hatch (significance not stated). While these two studies contradict each other, they demonstrate that the increase in activity of all three AOEs, so consistently found in mammalian foetuses, is not observed in this oviparous species. The different birthing strategy is associated with a different set of environmental conditions and appears to be associated with a different prenatal developmental pattern of AOE activity.

Similarly, the lungs of metamorphosing anurans are exposed to atmospheric oxygen tensions much earlier relative to development than mammalian embryos, and also than embryos of egg-laying vertebrates. Burggren and West (1982) demonstrated that lungs are present early in development in bullfrog (Rana catesbeiana) larvae (stage IV-V, according to the staging system of Taylor and Kollros (1946); this equates to about stage 26 of the staging system of Gosner (1960), which is used in the present study. Furthermore, larvae at all stages have been observed to air-breathe (Crowder et al., 1998). However, the lung does not become functional, in terms of oxygen uptake, until the late premetamorphic stages (stages XVI-XIX; approx. stage 36-40) (Burggren and West, 1982). The pulmonary gas-exchange area, the amount of pulmonary surfactant and static lung compliance, all increase as the tadpole finally metamorphoses into a froglet (Dupre et al., 1985; Oguchi et al., 1994). The activity of antioxidant enzymes in the lung of animals undergoing metamorphosis has not been described. However, the activity of whole body SOD has been found to increase during larval development in the anurans Discoglossus pictus and Rana ridibunda (Barja de Quiroga and Gutierrez, 1984). Furthermore, the activity of catalase increases during development in a number of organs (liver, kidney and pancreas) in the bullfrog Rana catesbeiana, but did not change in the African clawed toad Xenopus laevis (Dauca et al., 1982).

The present study examines the normal development of three antioxidant enzymes, catalase, SOD and GPx, in the perinatal lung of two oviparous species, the chicken (Gallus gallus) and the bearded dragon lizard (Pogona vitticeps), as well as in the metamorphosing lung of an Australian burrowing frog Limnodynastes terraereginae (Anura: Myobatrachidae: Limnodynastinae). We hypothesise that the activity of the antioxidant enzymes will be elevated much earlier relative to development in both oviparous and metamorphosing vertebrates, compared with mammalian embryos. In addition, we examined the lability of the AOEs in the embryonic chicken lung in response to a decrease in environmental oxygen, to test the hypothesis that the antioxidant enzymes are responsive to environmental oxygen.

\section{Materials and Methods}

Animals

Gravid bearded dragons Pogona vitticeps (Ahl, 1926) were collected from the Flinders Ranges, South Australia, in October 1998, after mating and before oviposition. Mothers 
were housed in cages $(1.0 \mathrm{~m} \times 0.65 \mathrm{~m} \times 0.45 \mathrm{~m})$ and were fed fruit, vegetables and cockroaches. They were provided with calcium and vitamin supplements and had access to water ad libitum. Clutches of 15-20 eggs were laid upon damp sphagnum moss. The eggs were removed immediately after laying, marked and placed in the same orientation as at oviposition in plastic boxes containing fine vermiculite (approx. $5 \mathrm{~mm}$ particles) with a gravimetric water content maintained at $1 \mathrm{~g} \mathrm{~g}^{-1}$ dry mass, resulting in a water potential of $>-200 \mathrm{kPa}$. Boxes were stored in a constant temperature cabinet at $29^{\circ} \mathrm{C}$ (Johnston et al., 2000).

Fertilized eggs from the chicken Gallus gallus L. were obtained from a commercial supplier (Globe Derby Poultry, Adelaide, South Australia) and randomly allocated to one of two groups. One group was incubated under normoxic conditions for the duration of incubation. The other group was incubated first under normoxic conditions and then transferred to hypoxic conditions (17.7 $\pm 0.3 \%$ atmospheric oxygen) at day 10 of incubation. All eggs were incubated in a Bellsouth 100 electronic incubator equipped with a Bellsouth 100AT automatic turner (Bellsouth, Narre Warren, Victoria, Australia) at $39^{\circ} \mathrm{C}$.

Spawn of Limnodynastes terraereginae Fry, 1915 was collected from a dam in Forestry Block 245, Atherton Tablelands, Queensland, Australia ( $\left.17^{\circ} 36^{\prime} 4^{\prime \prime} \mathrm{S}, 145^{\circ} 27^{\prime} 44^{\prime \prime} \mathrm{E}\right)$ on 20 January 1999. Tadpoles were reared in aerated water at ambient temperature before being transported to the laboratory at Adelaide University on 27 January 1999. There they were reared at $30 \pm 1^{\circ} \mathrm{C}$ in aerated, dechlorinated tapwater in glass aquaria $(25 \mathrm{~cm} \times 25 \mathrm{~cm} \times 8 \mathrm{~cm})$. Developing larvae (tadpoles) were fed lightly boiled, organically grown lettuce leaves supplemented with SERA bioflakes pond fish food. Water and food were changed daily.

\section{Experimental groups}

Bearded dragon embryos were killed at 50 days $(N=5)$ and at 55 days $(N=6)$ following oviposition as well as at pip $(N=6$; mean age at pipping $=57.5 \pm 1.1$ days $)$ and at hatch $(N=6$; mean age $=59 \pm 0.9$ days), by an overdose of sodium pentobarbitone injected intraperitoneally. Lungs were excised and stored at $-80{ }^{\circ} \mathrm{C}$ until analysis. Due to the small size of the embryos, it was not possible to sample lungs any earlier.

Chicken embryos were killed with an overdose of sodium pentobarbitone injected intraperitoneally on days 14, 16 and 18 of incubation, at pip (20 days normoxia, 19 days hypoxia) and at hatch (21 days normoxia, 20 days hypoxia) (Table 1). Lung tissue was stored at $-80^{\circ} \mathrm{C}$ until analysis.

Tadpoles were staged according to the staging system of Gosner (1960). These stages are represented by arabic numerals. References to developmental stages in the literature are from the system of Taylor and Kollros (1946) and are given as roman numerals. In this study, tadpoles from stage 30 to stage $46(N=30-40$ for each developmental stage) were used between March and June of 1999. Larvae of stages 30-40 were killed by decapitation. Premetamorphs at stages $41-45$ and metamorphs (stage 46) were killed with an overdose of
Table 1. Oxygen and carbon dioxide concentrations in the incubator containing chicken eggs under normoxic and hypoxic conditions

\begin{tabular}{|c|c|c|c|c|c|}
\hline \multirow{2}{*}{$\begin{array}{l}\text { Days of } \\
\text { incubation }\end{array}$} & \multirow{2}{*}{$\begin{array}{l}\text { Number } \\
\text { of eggs }\end{array}$} & \multicolumn{2}{|c|}{ Oxygen $(\%)$} & \multicolumn{2}{|c|}{ Carbon dioxide $(\%)$} \\
\hline & & Normoxia & Hypoxia & Normoxia & Hypoxia \\
\hline 1 & 40 & \multicolumn{2}{|c|}{20.9} & \multicolumn{2}{|c|}{0.04} \\
\hline 3 & 40 & \multicolumn{2}{|c|}{20.9} & \multicolumn{2}{|c|}{0.05} \\
\hline 5 & 40 & \multicolumn{2}{|c|}{20.9} & \multicolumn{2}{|c|}{0.07} \\
\hline 7 & 40 & \multicolumn{2}{|c|}{20.8} & \multicolumn{2}{|c|}{0.08} \\
\hline 9 & 40 & \multicolumn{2}{|c|}{20.8} & \multicolumn{2}{|c|}{0.014} \\
\hline 10 & 40 & 20.7 & 17.7 & 0.015 & 0.090 \\
\hline 11 & 40 & 20.7 & 17.5 & 0.017 & 0.011 \\
\hline 12 & 40 & 20.5 & 17.5 & 0.029 & 0.016 \\
\hline 13 & 40 & 20.4 & 18.0 & 0.400 & 0.031 \\
\hline 14 & 40 & 20.2 & 17.9 & 0.510 & 0.600 \\
\hline 15 & 33 & 20.4 & 17.5 & 0.390 & 0.780 \\
\hline 16 & 33 & 20.4 & 17.6 & 0.430 & 0.098 \\
\hline 17 & 25 & 20.5 & 17.7 & 0.280 & 0.066 \\
\hline 18 & 25 & 20.5 & 17.7 & 0.330 & 0.035 \\
\hline 19 & 18 & 20.9 & 17.8 & 0.040 & 0.036 \\
\hline 20 & 18 & 20.9 & 18.0 & 0.040 & 0.021 \\
\hline 21 & 11 & 20.9 & - & 0.040 & - \\
\hline
\end{tabular}

Measurements on days 14, 16, 18, 19 and 20 were taken before the incubator was opened to remove eggs.

pentobarbitone sodium injected intraperitoneally. The abdominal cavity was opened and lungs excised under a Wild M8 dissecting microscope. Lung samples were collected into precooled Eppendorf tubes and kept at $-80{ }^{\circ} \mathrm{C}$ prior to tissue processing.

In this study we did not correct for the antioxidant activity contained in blood, because the blood perfusing the lungs represents an important contribution to the overall pulmonary antioxidant defence system. In order to diminish variations in the extent of perfusion between samples of the same experimental group, and also between groups, we took care to excise lungs at an identical time and in an identical manner in each animal. Hence, lungs were removed after respiratory movements had ceased, but the heart was still beating. Furthermore, no major vessels or organs were cut, thereby standardising the level of blood loss.

\section{Hypoxia protocol}

A state of chronic hypoxia was induced by decreasing the oxygen content of the air within the incubator to $17.7 \pm 0.3 \%$ (Table 1). This level of hypoxia is considered to be relatively mild, and physiologically more relevant than other protocols which have induced hypoxia using $10 \% \mathrm{O}_{2}$. Hypoxia treatment began at day 10 of incubation and continued until the day the animal was killed $(14,16,18,19$ or 20 days) (Table 1). The gas was supplied by Douglas bags inflated with $17 \%$ oxygen in nitrogen and sucked through the incubator by a pump (flow rate $50 \mathrm{ml} \mathrm{min}^{-1}$ ) and was continuously monitored by an oxygen analyser. This method gave a very slow airflow over the eggs and any holes in the incubator were sealed to minimise 


\section{A. P. Starrs and others}

exchange with atmospheric gases. The incubator was only opened to obtain eggs on days $14,16,18$ and pip (19 days), and otherwise remained sealed. The increase in $\mathrm{CO}_{2}$ recorded around days $13-16$ is probably due to the gradual accumulation of respiratory gases from a large number of eggs in a sealed incubator. As the eggs were sampled, thereby reducing the number and opening the incubator, the $\mathrm{CO}_{2}$ decreased, but throughout incubation, the concentration of $\mathrm{CO}_{2}$ never exceeded $0.8 \%$. This concentration of $\mathrm{CO}_{2}$ is unlikely to affect respiration.

\section{Tissue preparation}

For bearded dragons and chickens, individual samples of lung tissue were used from each animal. In the case of frogs, lungs from 30-40 tadpoles in each developmental stage were weighed and then combined to give $50 \mathrm{mg}$ lung tissue per sample. This resulted in between 1 and 3 samples per developmental stage. Samples of frozen lung tissue were rapidly weighed in precooled Eppendorf tubes and cut vigorously for 2-3 min on ice with fine scissors in 1:20 w/v of ice-cold $10 \mathrm{mmoll}^{-1}$ potassium phosphate buffer ( $\mathrm{pH} 7.4$, supplemented with $30 \mathrm{mmoll}^{-1} \mathrm{KCl}$ and $1 \mathrm{mmoll}^{-1}$ EDTA). The fine tissue suspension was sonicated (MSE sonicator equipped with an exponential probe, $20 \mathrm{kHz}$, medium power, amplitude $16 \mu \mathrm{m})$ on ice for a total of $45 \mathrm{~s}(1 \mathrm{~s}$ burst $/ 4 \mathrm{~s}$ rest mode) for chicken and bearded dragon lung samples, and for a total of $30 \mathrm{~s}$ ( $1 \mathrm{~s}$ burst $/ 4 \mathrm{~s}$ rest mode) for frog lung tissue (Del Maestro and McDonald, 1985). Ice was replaced half-way through the sonication of each sample to prevent significant heating of the homogenate. The homogenate was centrifuged $(12,000 \mathrm{~g}, 5 \mathrm{~min})$ and the supernatant divided into four portions for SOD, catalase, GPx and protein assays and kept at $-80^{\circ} \mathrm{C}$ prior to biochemical analyses.

\section{AOE activity analyses}

Purified standards for catalase (from bovine liver; EC 1.11.1.6), SOD (from bovine erythrocytes; EC 1.15.1.1) and GPx (from bovine erythrocytes; EC 1.11.1.9) were purchased from Sigma Chemicals. All other chemicals were of analytical grade or higher. The activities of SOD, catalase and GPx were analysed using standard spectrophotometric assays performed with a double-beam spectrophotometer (Hitachi-U 2000) equipped with a constant temperature chamber.

Total SOD activity was measured by monitoring the SODinduced inhibition of cytochrome $c$ reduction by the superoxide radical generated in a xanthine/xanthine oxidase system (McCord and Fridovich, 1969). Briefly, samples of supernatant $(50-100 \mu \mathrm{l})$ were added to an assay mixture containing $50 \mathrm{mmol}^{-1}$ potassium phosphate buffer, $(\mathrm{pH} 7.8), 0.1 \mathrm{mmoll}^{-1}$ EDTA, $0.01 \mathrm{mmoll}^{-1}$ cytochrome $c, 0.05 \mathrm{mmoll}^{-1}$ xanthine and 0.005 units xanthine oxidase in a final volume of $3 \mathrm{ml}$. The rate of increase in absorbance was continuously recorded spectrophotometrically at $550 \mathrm{~nm}$ and $25^{\circ} \mathrm{C}$ for $5 \mathrm{~min}$ and the maximum linear rate was used to calculate the activity of SOD. One unit of SOD was defined as the quantity of SOD required to produce $50 \%$ inhibition of the rate of reduction of cytochrome $c$ in the system described above.

Catalase activity was determined by measuring the rate of $\mathrm{H}_{2} \mathrm{O}_{2}\left(10 \mathrm{mmol}^{-1}\right)$ decomposition in $50 \mathrm{mmol}^{-1}$ potassium phosphate buffer, (pH 7.0), at $240 \mathrm{~nm}$ and $25^{\circ} \mathrm{C}$ (Aebi, 1974; Claiborne, 1985). The activity was calculated using the molar extinction coefficient of $0.0436\left(\mathrm{mmol} \mathrm{l}^{-1}\right)^{-1} \mathrm{~cm}^{-1}$ (Aebi, 1974).

Total GPx activity was determined using an indirect, coupled enzyme method (Paglia and Valentine, 1967) in a modification described by Gunzler and Flohe (1985). GPx catalyses the glutathione-dependent reduction of hydroperoxides where reduced glutathione (GSH) is converted to its oxidised form (GSSG). In this method, GSSG is continuously reduced to GSH by NADPH in the presence of glutathione reductase. NADPH is continuously oxidised to NADP while the concentration of GSH is maintained constant. The amount of GPx in the sample is proportional to the amount of NADPH oxidised per unit time. In this study, the rate of NADPH consumption was continuously monitored spectrophotometrically at $340 \mathrm{~nm}$ and $37^{\circ} \mathrm{C}$ and used to calculate the amount of GPx.

Briefly, $100 \mu \mathrm{l}$ of supernatant was added to $400 \mu \mathrm{l}$ of $50 \mathrm{mmol}^{-1}$ potassium phosphate buffer, $\mathrm{pH} 7.0$, containing $1 \mathrm{mmoll}^{-1}$ EDTA and $100 \mu \mathrm{l}$ of $10 \mathrm{mmoll}^{-1}$ sodium azide, followed by $100 \mu \mathrm{l}$ of glutathione reductase $\left(2.4 \mathrm{U} \mathrm{ml}^{-1}\right)$ and $100 \mu \mathrm{l}$ of $10 \mathrm{mmoll}^{-1} \mathrm{GSH}$. The mixture was incubated for 10 min at $37^{\circ} \mathrm{C}$ and $100 \mu \mathrm{l}$ of $1.5 \mathrm{mmoll}^{-1} \mathrm{NADPH}$ in $0.1 \%$ $\mathrm{NaHCO}_{3}$ was added. The solution was mixed with a cuvette stirrer and the hydroperoxide-independent NADPH consumption was recorded at $340 \mathrm{~nm}$ and $37^{\circ} \mathrm{C}$ for $5 \mathrm{~min}$. $100 \mu \mathrm{l}$ of $1.5 \mathrm{mmol}^{-1} \mathrm{H}_{2} \mathrm{O}_{2}$ was then added, mixed and the decrease in absorbance was recorded for another $5 \mathrm{~min}$.

Sodium azide was added to inhibit catalase. One unit of GPx is defined as the amount of GPx causing the oxidation of

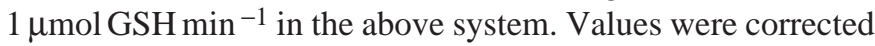
for the hydroperoxide-independent decomposition of $\beta$ NADPH as well as for the non-enzymatic oxidation of GSH.

Total protein content was determined using the Coomassie Brilliant Blue G-250 method (Lott et al., 1983). The absorbance of standard Coomassie Brilliant Blue solution was measured at $465 \mathrm{~nm}$ and $595 \mathrm{~nm}$ and the ratio of the absorbances was compared with a standard curve prepared using bovine serum albumin.

\section{Data analysis}

The activity of each enzyme was calculated in units $\mathrm{ml}^{-1}$ and standardised against $\mathrm{mg}$ protein $\mathrm{ml}^{-1}$. For each time point, activities are represented as the mean \pm S.E.M. units $\mathrm{mg}^{-1}$ protein. For chickens and lizards, differences between stages were determined using a Student's $t$-test, with a Bonferroni correction for multiple $t$-tests. Hypoxia and normoxia treatments were compared using a Student's $t$-test with significance taken as $P<0.05$. For the frogs, patterns of antioxidant development are illustrated with the simplest significant line of best fit through the individual points. 


\section{Results}

Pogona vitticeps

\section{Catalase}

Catalase activity increased throughout incubation from day 50 until pip and hatch in a linear fashion (Fig. 1A). Activity at both pip and hatch were significantly elevated above levels at day 50 ( $t=-2.31$, d.f. $=9, P=0.023$; $t=-3.58$, d.f. $=10, P=0.002$, respectively). Catalase activity of hatchlings was significantly elevated over adult levels ( $t=4.63$, d.f. $=11, P=0.0003$ ), indicating that, at some point after hatch, activity decreases.

\section{$S O D$}

SOD activity displayed a different pattern to that of catalase. SOD decreased from day 50 through to pip in a linear manner (Fig. 1B). Activity at pip was significantly lower than at day 50 ( $t=2.23$, d.f. $=9, P=0.026)$. SOD activity in the lung of adult lizards was similar to that of pipping and hatchling lizards. significantly from day 18 to pip ( $t=2.7$, d.f. $=11, P=0.01$ ). Under hypoxic conditions, SOD activity remained constant throughout incubation (Fig. 2B). Activity did not differ between hypoxic and normoxic embryos at any time point.

\section{GPx activity}

GPx activity in the prenatal normoxic lung demonstrated a sharp increase between day 16 and day 18 ( $t=-3.36$, d.f. $=11$, $P=0.0032$ ) and remained constant until hatch (Fig. 2C). GPx activity demonstrated the greatest differences between normoxic and hypoxic conditions of any of the three enzymes (Fig. 2C). Under hypoxic conditions, GPx activity was constant from day 14 to pip, and then increased significantly from pip to hatch ( $t=-4.11$, d.f. $=7, P=0.022)$. GPx activity in hypoxic chicken embryos was elevated above normoxic values for day $14(t=-1.92$, d.f. $=11, P=0.040)$, day 16 $(t=-3.05$, d.f. $=11, P=0.0055)$ and hatch $(t=-2.28$, d.f. $=11$, $P=0.022$ ).

\section{$G P x$}

There were fewer data available for GPx activity because the lungs of Pogona vitticeps were very small. Only those samples with sufficient tissue homogenate left after the SOD and catalase analyses were measured for GPx. GPx remained fairly constant and low throughout late incubation (Fig. 1C). Due to small sample sizes $(N=1$ or 2 ) it was not possible to determine whether there was an increase in GPx activity upon hatching. Adult GPx activity was almost threefold higher than at any point during the perinatal period (Fig. 1C).

$$
\text { Gallus gallus }
$$

\section{Catalase activity}

Catalase activity in the normoxic chicken lung rose throughout incubation from day 14 until pip (20 days) (Fig. 2A). Activity at pip was significantly elevated above levels at day 14 ( $t=-5.83$, d.f. $=12, P=0.00004)$. Catalase activity in the normoxic lung fell significantly from pip to hatch $(t=4.65$, d.f. $=13, P=0.00024)$. In hypoxic embryos, pip and hatch occurred a day earlier than under normoxic conditions (Fig. 2). Under hypoxic conditions, catalase activity did not demonstrate a significant increase from day 14 to pip ( $t=-1.86$, d.f. $=8$, $P=0.49$ ). Furthermore, once a Bonferroni correction is applied, there was also no significant decrease from pip to hatch $(t=1.90$, d.f. $=7, P=0.049$ ) (Fig. 2A).

\section{SOD activity}

SOD activity in the normoxic embryo remained constant from day 14 to day 18 (Fig. 2B). After this period, activity fell
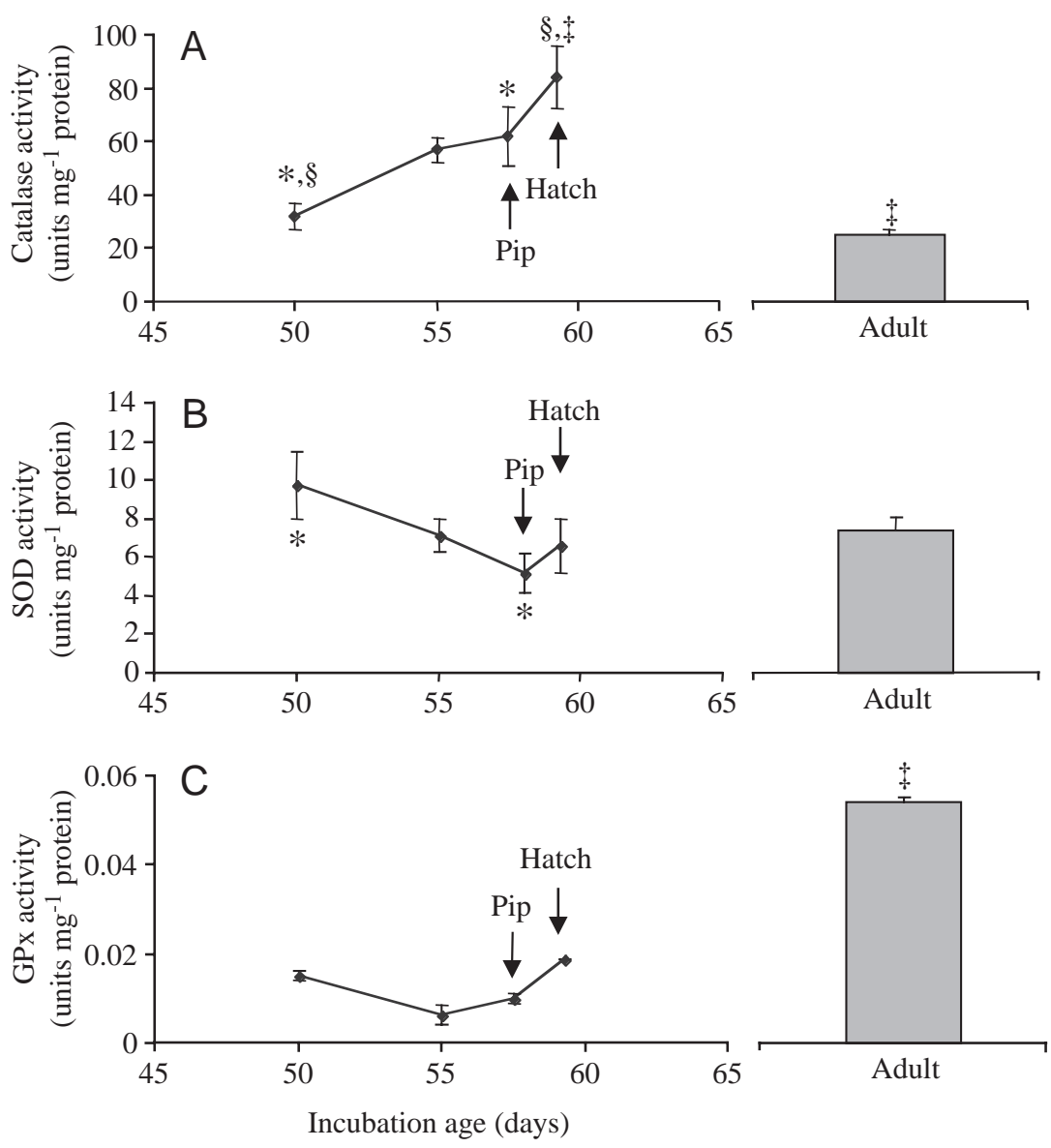

Fig. 1. Changes in the activity of antioxidant enzymes catalase (A), SOD (B) and GPx (C) in the lungs of the bearded dragon Pogona vitticeps during late development, at hatch and in the adult. Values are units $\mathrm{mg}^{-1}$ protein and are means \pm S.E.M. In all cases $N=4-6$, except in C, where 'pip' and 'hatch' data points are $N=2$ and $N=1$, respectively. Pairs of symbols $(*, \S$ and $\ddagger)$ indicate points that are significantly different from one another $(P<0.05)$. In $\mathrm{C}$, $\neq$ indicates that adult GPx activity is significantly higher than at any other stage of development. 


\section{Limnodynastes terraereginae}

Catalase activity in the lung tissue of Limnodynastes terraereginae larvae was present at early stages of larval development. The activity of this enzyme was relatively constant between stages 30 and 40 but increased exponentially thereafter (Fig. 3A). Both SOD and GPx activity were present in lung tissue of stage-30 tadpoles and did not change throughout metamorphosis (Fig. 3B and C).

\section{Discussion}

Numerous studies in mammals have established that the three AOEs (catalase, SOD, GPx) are regulated independently (Chen and Frank, 1993). Differential regulation of these enzymes is also evident in the two oviparous species and the metamorphosing frog examined in the present study. Whereas the developmental pattern of catalase activity is essentially similar in all three species, the other two enzymes demonstrate very varied patterns among the three species.

\section{Catalase activity}

In both oviparous species, catalase activity increased during the final $20 \%$ of incubation until pipping. The AOE activity for the two oviparous species must be viewed in the light of a progressive decrease in oxygen tension in venous blood of the developing embryo throughout late incubation. For example, in the chicken there is a decrease in oxygen tension $\left(\mathrm{P}_{\mathrm{O}_{2}}\right)$ of venous blood from $71.7 \mathrm{mmHg}$ at day 14 to $49.8 \mathrm{mmHg}$ at day $18 . P_{\mathrm{O}_{2}}$ then increases sharply at pipping to $82.9 \mathrm{mmHg}$ (Freeman and Misson, 1970). Hence, the increase in catalase activity prior to pipping occurs despite a decrease in $P_{\mathrm{O}_{2}}$ in the chicken lung. Furthermore, catalase activity decreased from pip to hatch (Fig. 2A), despite a sharp increase in blood $\mathrm{PO}_{2}$ at that time. This suggests that catalase does not respond to the changes in blood oxygen levels. Furthermore, hypoxia did not alter catalase activity in the chicken lung, thus it appears that catalase activity is not correlated with oxygen availability in the chicken lung. There is a similar pattern of change in catalase activity in the bearded dragon lung during late incubation through to pipping. However, blood $P_{\mathrm{O}_{2}}$ values in bearded dragon embryos are not known.

In tadpoles, pulmonary catalase activity remained relatively constant between stages 30 and 40, and thereafter there was an exponential increase in activity. Burggren and West (1982) determined that significant $\mathrm{O}_{2}$ uptake by the lungs in bullfrog (Rana catesbeiana) larvae does not occur until stage XVI (equivalent approximately to stage 36). Furthermore, stage XXII (equivalent approximately to stage 42) is a critical developmental stage during which bullfrog tadpoles switch from a preference for water $(95 \%)$ to a preference for land $(74 \%)$ (Crowder et al., 1998). It is interesting that the timing of this switch correlates exactly with the rapid increase in catalase activity. It is tempting to speculate that it is the sudden increase in oxygen uptake through increased dependence on air-breathing that is inducing catalase activity, suggesting that catalase in this species is responsive to environmental oxygen. However, without direct measurements of oxygen uptake in this particular species of frog, this conclusion remains speculative.

The increase in catalase activity during the final $20 \%$ of incubation is very similar to that reported for most mammals (Frank and Sosenko, 1987; Walther et al., 1991). Such a similar developmental pattern between widely divergent species would indicate that these biochemical changes are part of a general process of lung maturation and represent a normal 'preparation for birth'. This is further supported by the absence
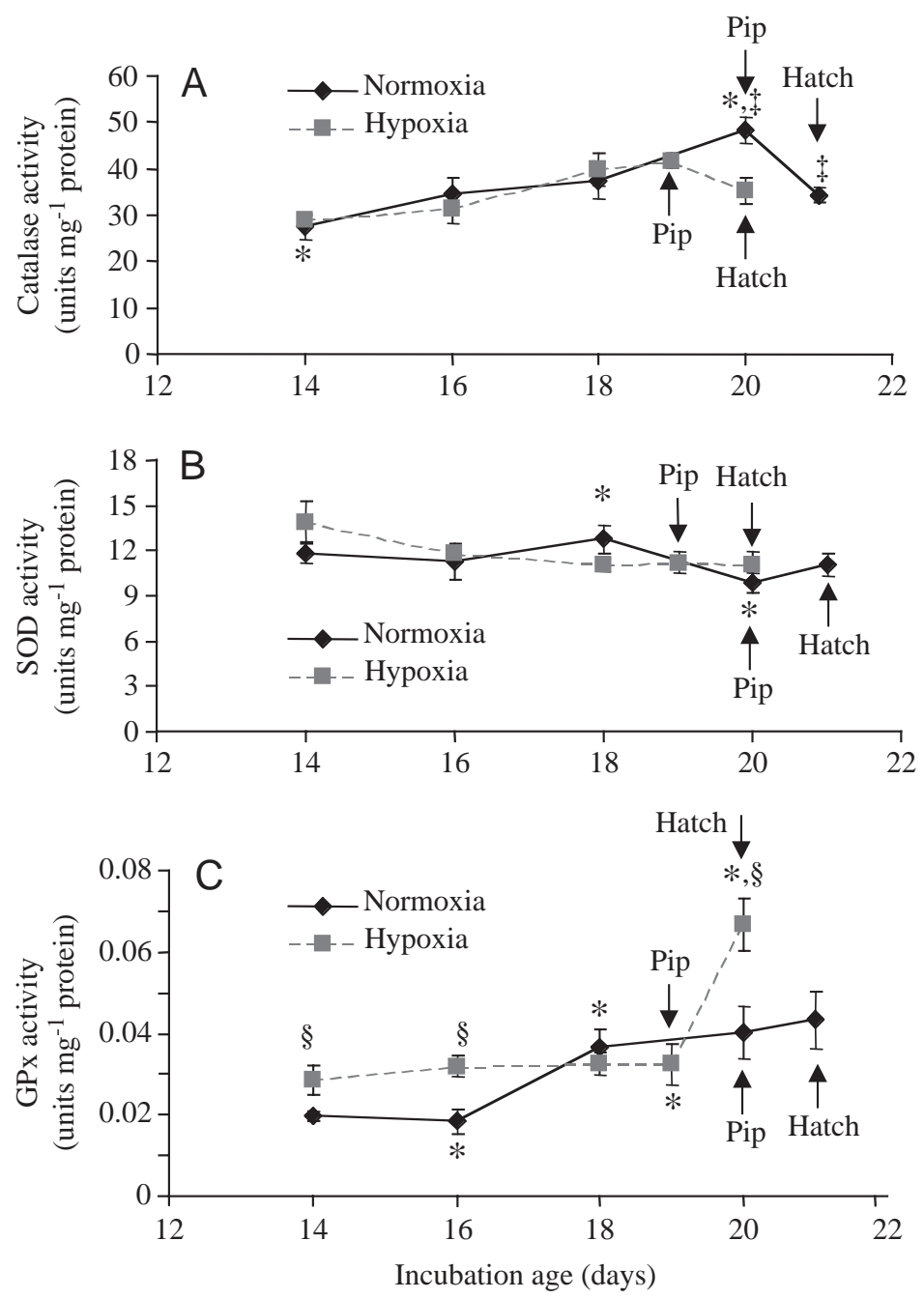

Fig. 2. Changes in the activity of antioxidant enzymes catalase (A), SOD (B) and GPx (C) in the lungs of the chicken Gallus gallus during late development and at hatch. Solid lines indicate changes under normoxic conditions and dashed lines indicate changes under hypoxic $\left(17 \% \mathrm{O}_{2}\right)$ conditions. Values are units $\mathrm{mg}^{-1}$ protein and are means \pm S.E.M. In all cases $N=4-6$. Pairs of symbols (* and $\ddagger$ ) within the same experimental group indicate points that are significantly different from one another $(P<0.05) . \S$ indicates points for which there is a significant difference in antioxidant enzyme activity between normoxic and hypoxic conditions. 
of an environmentally induced effect on the levels of this particular AOE in chickens. As the developmental pattern of catalase activity is not subject either to birth strategy or to environmental influences, it is probably predetermined. A possible mechanism for this programming may be related to the increase in reactive oxygen species that occurs in all tissues during late development as a result of the increased metabolism associated with differentiation and growth (Frank et al., 1996). It has recently been proposed that the concomitant developmental increase in reactive oxygen species, which form the substrates for the AOEs, may represent the primary controlling mechanism for AOE induction (Frank et al., 1996).
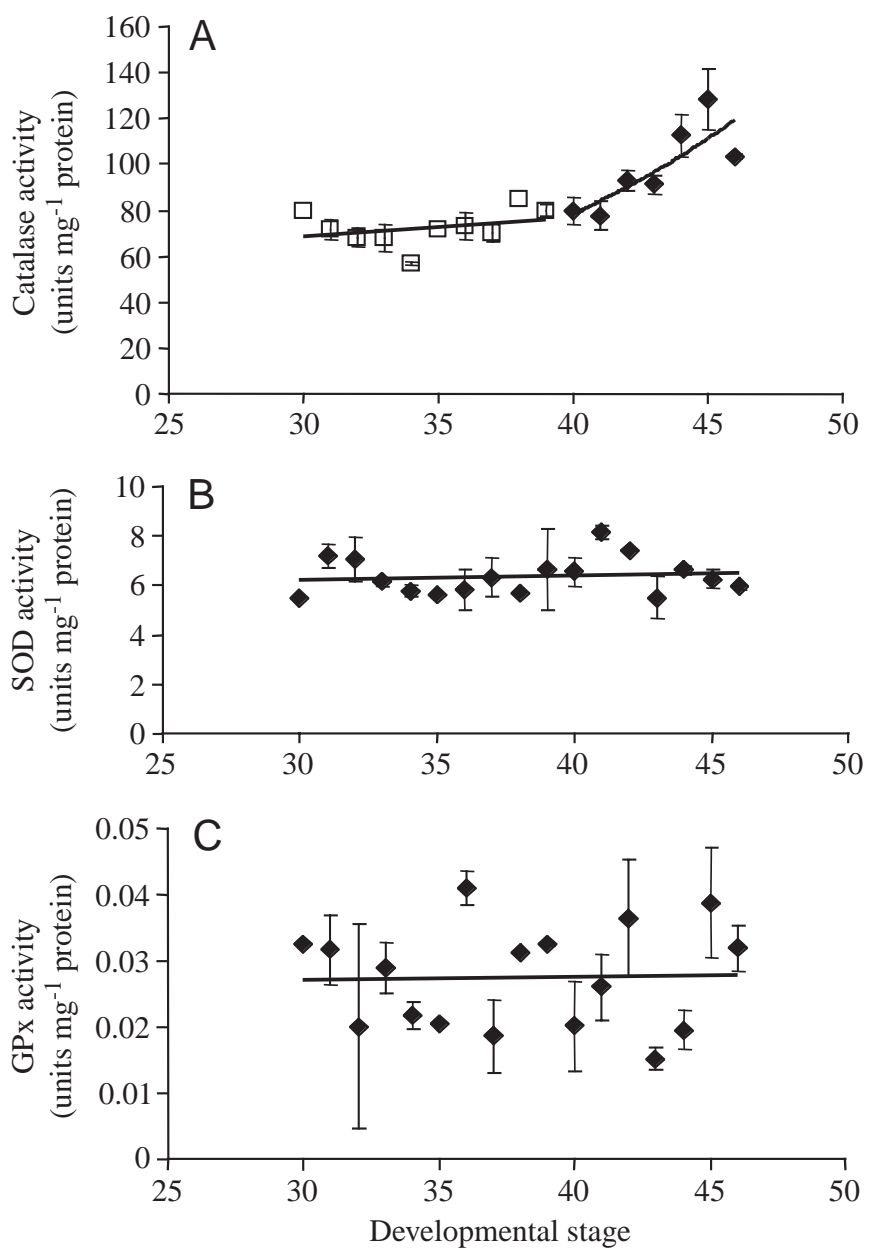

Fig. 3. Changes in the activity of antioxidant enzymes expressed as units $\mathrm{mg}^{-1}$ protein in lung tissue of frogs at developmental stages 30-46. Lungs from 10-30 animals were pooled for each sample. Values are means, means \pm range or means \pm S.E.M., depending on the number of measurements per stage ( $N=1$ for stages $30,35,38$; $N=2$ for stages $32,34,42 ; N=3$ for all other stages). Catalase activity (A) remained unchanged for stages 30-40 ( $y=0.8272 x+43.811$; $\left.r^{2}=0.0997 ; \quad N=10\right)$ and thereafter increased exponentially $\left(y=4.6563 \mathrm{e}^{0.0705 x} ; \quad r^{2}=0.7059 ; \quad N=7 ; \quad P<0.01\right)$. SOD activity (B) remained unchanged over the entire developmental period $\left(y=0.0175 x+5.7001 ; r^{2}=0.0137 ; N=17\right)$. GPx activity (C) remained unchanged over the entire developmental period $\left(y=5 \times 10^{-5} x+0.0255\right.$; $\left.r^{2}=0.0011, N=17\right)$.
The pattern of catalase development (an increase throughout incubation until pipping) in the present study resembles that presented in a previous study (Van Golde et al., 1988). In contrast, Surai found that catalase activity in chicken lung remained relatively unchanged throughout incubation from day 15 to day 19, with a small increase toward hatching (significance not stated) (Surai, 1999). It is unclear why the results of Surai should differ from those of Van Golde and coworkers (Van Golde et al., 1998) and the present study. Here, absolute values of catalase activity from developing chicken lungs are up to an order of magnitude greater than those measured in the earlier studies. Methodological differences between the present study and the previous measurements of AOEs in chicken lung (Surai, 1999; Van Golde et al., 1998) may contribute to the varied results. Sonication was used to disperse the cells in the present study, as opposed to homogenisation. In addition to releasing cytosolic enzymes, sonication also ruptures cellular organelles to release organelle-specific AOEs. As catalase is concentrated in peroxisomes, this may explain the dramatically greater values presented here for catalase activity.

\section{SOD activity}

The response of this enzyme to both natural and experimental oxygen tensions is difficult to interpret. In the lung of the bearded dragon, SOD activity continued to decrease throughout late development. While no change in SOD activity occurred between days 14 and 18 in the embryonic chicken lung, there was a significant decrease between days 18 and 19 (pip). Given the relatively longer total incubation period of bearded dragons (60 days), the decrease just prior to pipping in both dragons (day 50) and chickens (day 18) occurs at very similar stages during total incubation, i.e. at $83 \%$ and $85 \%$, respectively. Hence, the change in SOD activity under normoxic conditions is similar for both oviparous vertebrates, but directly opposite to that of mammals (Frank and Sosenko, 1987). Our data also indicate that SOD activity in the chicken is not responsive to environmental $P_{\mathrm{O}_{2}}$. First, despite the large increase in $P_{\mathrm{O}_{2}}$ leading up to pipping in the chicken (Freeman and Misson, 1970), there was a significant decrease in SOD activity. Secondly, during early incubation (days 14-16) there was no change in SOD activity, despite the greatest decrease in $P_{\mathrm{O}_{2}}$ at this stage (Freeman and Misson, 1970). Thirdly, despite the rapid increase in arterial and alveolar $P_{\mathrm{O}_{2}}$ upon hatching, there was no change in SOD activity following pipping in either species. Fourthly, SOD activity in chickens exposed to hypoxic conditions remained constant throughout late incubation (day 14-19), demonstrating no statistically significant difference between normoxic and hypoxic activity at any developmental age. However, hypoxia did abolish the decrease in SOD activity that was observed under normoxic conditions from day 18 to pip. Hence, hypoxia did cause a slight alteration in the SOD activity profile of embryonic chickens.

Unlike catalase, SOD data in the present study are very similar to those obtained by Surai (1999), where the activity of the enzyme was found in comparable levels and was lowest immediately prior to hatching. This contrasts with the results 
obtained by Van Golde and coworkers who found a peak in SOD activity on day 19 and values that were 8-10 times higher than the other studies (Van Golde et al., 1998). The reason for these differences again is unclear.

In tadpoles, SOD activity remained unchanged throughout metamorphosis from stage 30 to 46 . This indicates firstly, that SOD is present at mature activity levels in very immature lungs at stage 30, when the lungs are not yet functional in a respiratory sense. Secondly, the enzyme does not appear to be responsive to environmental oxygen, which increases with airbreathing at around stage 40 (see discussion on GPx activity of tadpoles).

\section{GPx activity}

The data for GPx activity of the bearded dragon need to be treated with caution as they consist of relatively few measurements due to the small amounts of lung tissue that we could isolate. However, in early development of the bearded dragon, GPx activity does not appear to change. Given the small sample sizes at pip and hatch, it was not possible to determine whether there is a significant increase in GPx activity upon air-breathing. It is striking, however, that the level of GPx activity in adult lung tissue is threefold greater than in hatchling bearded dragons. Given the fact that catalase demonstrates the opposite trend, i.e. it is present at greater levels in hatchlings than in adults, these results suggest that in the defence against $\mathrm{H}_{2} \mathrm{O}_{2}$, catalase may be the more important enzyme at parturition, whereas GPx may be the more important antioxidant enzyme in the adult.

The notable feature in the developmental pattern of GPx activity in the normoxic lung of Gallus gallus is the sharp increase from day 16 to day 18 . This increase occurred at a time when blood $\mathrm{PO}_{2}$ in the chicken embryo is low (Freeman and Misson, 1970), suggesting that activity of GPx is increasing, like that of catalase, in a genetically determined developmental pattern, in preparation for birth (Frank and Sosenko, 1987). However, in the hypoxic chicken lung, over the time frame examined, it appears that this increase was abolished, as GPX activity was elevated compared with normoxic chickens at days 14 and 16, and remained constant throughout late incubation. A possible explanation for the lack of an increase in GPX activity between days 16-18 in the hypoxic chicken lung is that the increase occurred earlier (prior to day 14), because the levels of GPx activity were already elevated at days 14 and 16 compared with normoxic chickens. This is supported by the fact that development was accelerated under hypoxic conditions (i.e. pip and hatch occurred one day earlier), and may point to a genetic mechanism that has been speeded up, i.e. the environment has a modulating influence. Further evidence that environmental oxygen is able to influence GPx activity comes from the dramatic (100\%) increase in GPx activity upon hatching in hypoxic chickens. This is the largest increase in any of the treatments and analyses. It is possible that this increase may be a result of the more pronounced relative hyperoxia that would occur in hypoxic chickens upon hatching, compared with normoxic chickens.
In tadpoles, GPx activity, like that of SOD, remained unchanged throughout metamorphosis. Hence, neither enzyme appears to be responsive to the environment, and both enzymes were present at mature activity levels in very immature lungs at stage 30. In normoxic water, stage I-XXI bullfrog (Rana catesbeiana) larvae (equivalent approximately to stages 20-41) breathe air regularly but infrequently (0.4-6.2 surfacings per hour). Earlier stages breathe more frequently than later stages. However, breathing at these stages is not required for oxygen. Burggren and West (1982) determined that significant $\mathrm{O}_{2}$ uptake by the lungs in $R$. catesbeiana does not occur until stage XVI (equivalent approximately to stage 36). Breathing in the early developmental stages may serve to promote lung development and prevent accumulation of fluid in the lung (Crowder et al., 1998). In terrestrial embryos, the lung is filled with fluid, which is necessary for lung growth and development. In aquatic larvae a fluid-filled lung would reduce buoyancy. Although the lungs of L. terraereginae at these early stages are unlikely to be functional for gas exchange, we did observe frequent gulping of air at the surface, and the lungs upon excision were usually inflated. Hence, the potential for oxygen radical damage is high. It is likely, therefore, that high levels of SOD and GPx are required at these early stages to protect the developing, epithelial lining from oxidative damage. Furthermore, as developing tadpoles are undergoing extensive organogenesis (Duellman and Trueb, 1986), the oxygen consumption of individual tissues will be high, as will be the levels of reactive oxygen species. As the level of oxygen consumption and hence the level of reactive oxygen species is one of the chief factors determining the level of AOE (DeHaan et al., 1994; Frank and Groseclose, 1984), this may further explain the elevated levels of AOE during very early development in tadpole larvae.

\section{Conclusions}

In all three species, catalase activity increased during late incubation, in a manner similar to that of mammals. Such a similar developmental pattern between widely divergent species indicates that these biochemical changes are part of a general process of lung maturation and represent a normal 'preparation for birth'. The data from both oviparous species suggests that catalase activity is unresponsive to $\mathrm{O}_{2}$ fluctuations, and continues to increase in readiness for hatching, thus supporting the theory of a genetically programmed increase. However, in tadpoles, there is some evidence that catalase activity may be induced by increases in oxygen tensions. The decrease in SOD activity during very late incubation in the two oviparous species, when venous $\mathrm{P}_{\mathrm{O}_{2}}$ is increasing, is opposite to the change observed in mammals. Furthermore, in tadpoles, SOD activity was elevated very early during development and did not change thereafter. The bulk of the data suggest that SOD activity is not responsive to environmental oxygen, indicating that it too may be largely genetically determined. Although under normoxic conditions GPx appears to be under genetic control, this enzyme demonstrates the greatest capacity for environmental 
responsiveness. For example, it appears that the overall effect of hypoxia is to accelerate development in the chicken (hatching a day earlier), and this acceleration is reflected in the developmental changes in GPx activity. However, in tadpoles, GPx activity was elevated very early during development and remained unchanged thereafter, suggesting a purely genetic mechanism without any environmental modulation. Given the widely divergent evolutionary origin and different selection pressures acting on these organisms, it is highly likely that the antioxidant enzymes have evolved different induction mechanisms and different levels of dependency on environmental variables in these species. Finally, it appears that the late developmental increase in AOE activity that occurs in most mammals is not as predictable for oviparous and metamorphosing non-mammals. Moreover, the antioxidant enzyme system in metamorphosing tadpoles is in place at a relatively much earlier stage than in other vertebrates.

The authors wish to thank Sonya Johnston for her assistance with the lizard surgery and for critically reviewing the manuscript. We thank Jonathan Codd and Lucy Sullivan for technical assistance, Rebecca Skinner and Simon Chamberlain for maintaining and feeding the tadpoles, and Keith McDonald, Graeme Watson and Nicholas Birks for assistance with spawn collection. We thank the Department of Genetics for the use of their sonicator. Lizards were collected under National Parks and Wildlife Permit no. K23449-05. Spawn was collected under a Queensland Department of Environment and Heritage permit (F1/000202/98/SAA). All animal surgery was performed under University of Adelaide Animal Ethics Committee Permits M/045/97 and S/30/99. This project was funded by an Australian Research Council (ARC) grant to C.B.D. and an ARC Research Fellowship to S.O.

\section{References}

Aebi, H. (1974). Catalase. In Methods of Enzymatic Analysis (eds. H. U. Bergmeyer and V. C. Weinheim), pp. 673-683. New York, London: Academic Press.

Barja de Quiroga, G. and Gutierrez, P. (1984). Superoxide dismutase during the development of two amphibian species and its role in hyperoxia tolerance. Mol. Physiol. 6, 221-232.

Burggren, W. W. and West, N. H. (1982). Changing respiratory importance of gills, lungs and skin during metamorphosis in the bullfrog Rana catesbeiana. Respir. Physiol. 47, 151-164.

Chen, Y. and Frank, L. (1993). Differential gene expression of antioxidant enzymes in the perinatal rat lung. Pediatr. Res. 34, 27-30.

Chiu, D. T. Y., Stults, F. H. and Tappel, A. L. (1976). Purification and properties of rat lung soluble glutathione peroxidase. Biochim. Biophys. Acta 445, 558-566.

Claiborne, A. (1985). Catalase activity. In CRC Handbook of Methods for Oxygen Radical Research (ed. R. A. Greenwald), pp. 283-284. Florida: Boca Raton.

Crowder, W. C., Nie, M. and Ultsch, G. R. (1998). Oxygen uptake in bullfrog tadpoles (Rana catesbeiana). J. Exp. Zool. 280, 121-134.

Dauca, M., Calvert, R., Ménard, D., Hugon, J. S. and Hourdry, J. (1982). Development of peroxisomes in amphibians. II. Cytochemical and biochemical studies on the liver, kidney, and pancreas. J. Exp. Zool. 223, $57-65$.

DeHaan, J. B., Tymms, M. J., Cristiano, F. and Kola, I. (1994). Expression of copper/zinc superoxide dismutase and glutathione peroxidase in organs of developing mouse embryos, fetuses, and neonates. Pediatr. Res. 35, $188-195$.

Del Maestro, R. F. and McDonald, W. (1985). Oxidative enzymes in tissue homogenates. In CRC Handbook of Methods for Oxygen Radical Research (ed. R. A. Greenwald), pp. 291-296. Florida: Boca Raton.

Duellman, W. E. and Trueb, L. (1986). Eggs and Development. In Biology of Amphibians, pp. 109-139. New York: McGraw-Hill Book Company.

Dupré, R. K., Taylor, R. F. and Frazier, D. T. (1985). Static lung compliance during the development of the bullfrog, Rana catesbeiana. Respir. Physiol. 59, 231-238.

Frank, L. and Groseclose, E. E. (1984). Preparation for birth into an $\mathrm{O}_{2}$-rich environment: The antioxidant enzymes in the developing rabbit lung. Pediatr. Res. 18, 240-243.

Frank, L. and Massaro, D. (1980). Oxygen toxicity. Am. J. Med. 69, 117-126.

Frank, L., Price, L. T. and Whitney, P. L. (1996). Possible mechanism for late gestational development of the antioxidant enzymes in the fetal rat lung. Biol. Neonate 70, 116-127.

Frank, L. and Sosenko, I. R. S. (1987). Prenatal development of lung antioxidant enzymes in four species. J. Pediatr. 110, 106-109.

Freeman, B. M. and Misson, B. H. (1970). pH, $P_{\mathrm{O}_{2}}$ and $P_{\mathrm{CO}_{2}}$ of blood from the foetus and neonate of Gallus domesticus. Comp. Biochem. Physiol. 33, $763-772$.

Fridovich, I. (1978). The biology of oxygen radicals. Science 201, 875-879.

Fridovich, I. and Freeman, B. (1986). Antioxidant defenses in the lung. Ann. Rev. Physiol. 48, 693-702.

Gerdin, E., Tyden, O. and Eriksson, U. J. (1985). The development of antioxidant enzymatic defense in the perinatal rat lung: Activities of superoxide dismutase, glutathione peroxidase, and catalase. Pediatr. Res. 19, 687-690.

Gosner, K. L. (1960). A simplified table for staging anuran embryos and larvae with notes on identification. Herpetologica 16, 183-190.

Gunzler, W. A. and Flohe, L. (1985). Glutathione peroxidase. In CRC Handbook of Methods for Oxygen Radical Research (ed. R. A. Greenwald), pp. 285-290. Boca Raton, Florida: CRC Press.

Johnston, S. D., Orgeig, S., Lopatko, O. V. and Daniels, C. B. (2000). Development of the pulmonary surfactant system in two oviparous vertebrates. Am. J. Physiol. 278, R486-R493.

Lott, J. A., Stephan, V. A. and Pritchard Jr., K. A. (1983). Evaluation of the Coomassie Brilliant Blue G-250 method for urinary protein. Clin. Chem. 29, 1946-1950.

McCord, J. M. and Fridovich, I. (1969). An enzymic function for erythrocuprein (hemocuprein). J. Biol. Chem. 244, 6049-6055.

Oguchi, A., Mita, M., Ohkawa, M., Kawamura, K. and Kikuyama, S. (1994). Analysis of lung surfactant in the metamorphosing bullfrog (Rana catesbeiana). J. Exp. Zool. 269, 515-521.

Paglia, D. E. and Valentine, W. N. (1967). Studies on the quantitative and qualitative characterization of erythrocyte glutathione peroxidase. J. Lab. Clin. Med. 70, 158-169.

Rickett, G. M. W. and Kelly, F. J. (1990). Developmental expression of antioxidant enzymes in guinea pig lung and liver. Development 108, 331-336.

Starck, J. M. (1998). Structural variants and invariants in avian embryonic and postnatal development. In Avian Growth and Development (ed. J. M. Starck and R. E. Ricklefs), pp. 59-88. New York: Oxford University Press.

Surai, P. F. (1999). Tissue-specific changes in the activities of antioxidant enzymes during the development of the chicken embryo. Br. Poult. Sci. $\mathbf{4 0}$, $397-405$.

Tanswell, A. K. and Freeman, B. A. (1984). Pulmonary antioxidant enzyme maturation in the fetal and neonatal rat. I. Developmental profiles. Pediatr. Res. 18, 584-587.

Taylor, A. C. and Kollros, J. (1946). Stages in the normal development of Rana pipiens larvae. Anat. Rec. 94, 7-24.

Van Golde, J. C., Borm, P. J., Wolfs, M. C., Rhijnsburger, E. H. and Blanco, C. E. (1998). Induction of antioxidant enzyme activity by hyperoxia $\left(60 \% \mathrm{O}_{2}\right)$ in the developing chick embryo. J. Physiol. 509, $289-296$.

Van Golde, L. M. G., Batenburg, J. J. and Robertson, B. (1988). The pulmonary surfactant system: biochemical aspects and functional significance. Physiol. Rev. 68, 374-455.

Walther, F. J., Wade, A. B., Warburton, D. and Forman, H. J. (1991). Ontogeny of antioxidant enzymes in the fetal lamb lung. Exp. Lung Res. 17, 39-45. 